\title{
Research on Application of remote video monitoring system in construction site management
}

\author{
Lei Shi ${ }^{1, a}$ and Xueliang $\mathrm{Hou}^{2, \mathrm{~b}}$ \\ ${ }^{1,2}$ Institute of Construction Technology and Management, North China Electric Power University, \\ Beijing 102206, China \\ aslhh123@126.com, bhou-xl@163.com
}

Keywords: Construction site; Monitoring system; Engineering management

\begin{abstract}
Compared with the traditional project management model, the installation and use of the remote video monitoring system in the construction site is a bold attempt and innovation. In order to strengthen the quality and safety production management, the video monitoring system and the Internet technology are applied to the project site to realize the real-time dynamic remote monitoring of the whole construction process. Therefore, the analysis and Research on the structure, working principle and function of the remote video surveillance system will help to improve the management level and effect of the project construction.
\end{abstract}

\section{Introduction}

At present, the amount of construction in our country has ranked first in the world. However, compared with the developed countries, there is still a big gap between the application of the project management level and the information technology. With the continuous improvement of the socialist economy, the rapid development of all walks of life and the continual expansion of construction projects, the ensuing problems of quality and safety have also become more serious. With the continuous emergence of large and extra-large construction projects, site construction is becoming more and more complicated. At the same time, joint construction are becoming more and more common. Also the number of site operations is getting larger and larger and more and more workers are employed. All these bring severe challenges to the dispatching, safety and quality management of the construction site. How to strengthen the safety management of construction site, reduce the frequency of occurrence of accidents, eliminate all kinds of illegal operations and civilized construction, control the key process effectively and Improve the quality of construction always was the focus of attention of construction enterprises and government[1].

With the continuous development of computer application technology, Internet technology, mobile communication technology and monitoring technology, the application of remote video surveillance is becoming more and more widely. The remote video surveillance system is applied to the production management, engineering quality, safety and civilized construction, environmental protection supervision work in the construction project, which is an important means of construction site management under the current situation.

\section{Construction of Remote Site Monitoring System}

\subsection{System introduction}

The system is composed of two parts: the project sit monitoring system and the remote monitoring center system. The monitoring system of the project site is the premise of remote monitoring. The main function of the project site monitoring system is to collect the video information of the construction site, and transmit it to the receiving end through the wireless communication technology so as to realize the monitoring of the whole construction schedule and key parts. At the same time, the information is stored, and when the quality problems, security problems and disputes occur, it can be historically searched and traced. The monitoring center 
monitoring system can share the monitoring data of each project through the network communication technology to realize the function of remote monitoring of all the projects.

\subsection{System working principle}

The system structure is shown in Figure 1, The video signal collected by the field camera is digitized and compressed, then sent it to the local video surveillance through the wired or 5.8G wireless network, so you can see the scene of the camera project scene photographed in the monitoring room. The control center communicates with the engineering site through the INTERNET or the private network, and monitors all the engineering sites within the jurisdiction.

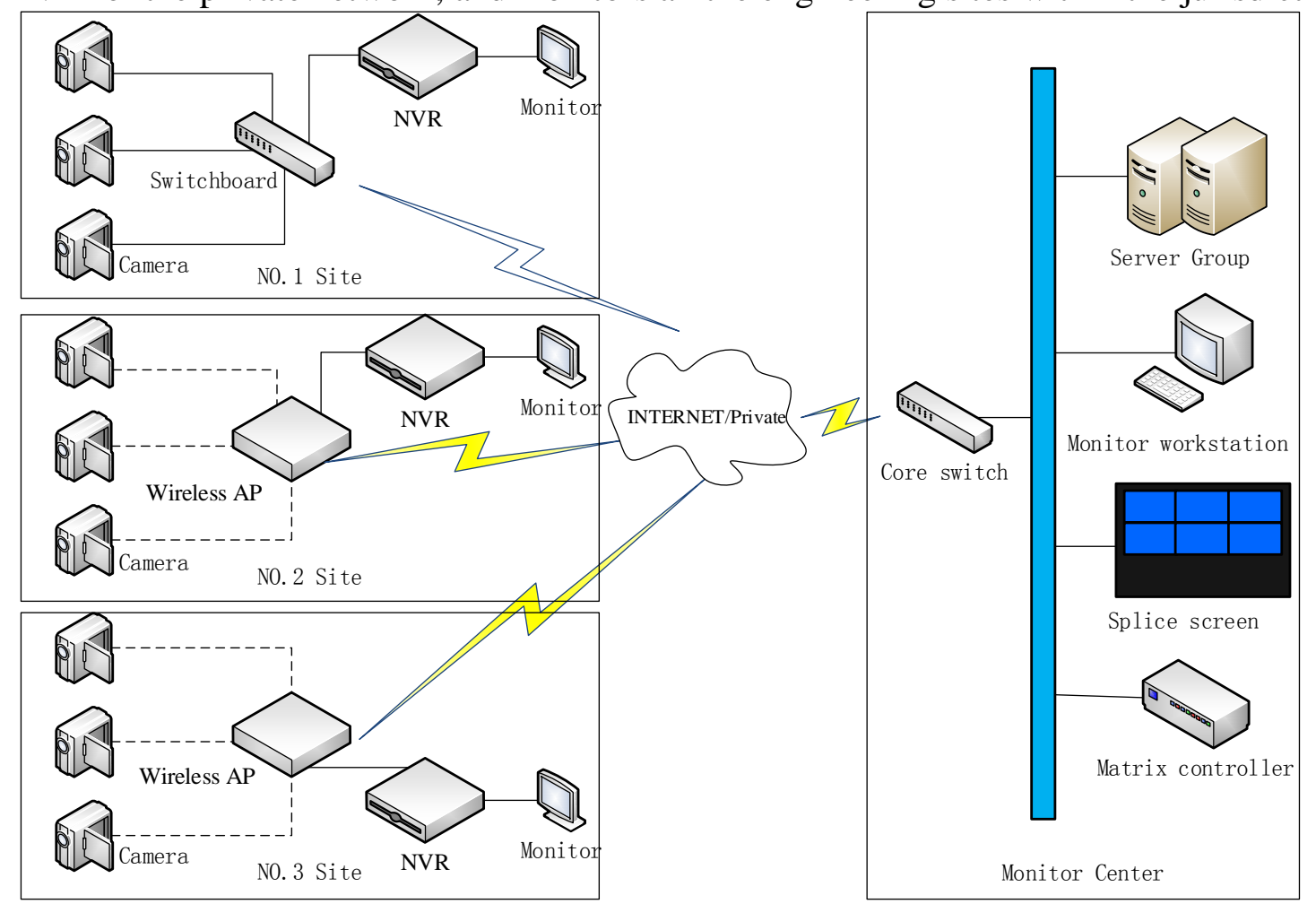

Fig.1. The system structure

\subsection{Hardware structure}

The hardware of the system is mainly composed of camera, 5.8G wireless AP, switch, NVR, server group, client PC and so on.

\subsubsection{Camera}

The camera is the front part of the monitoring system and the eye of the whole system".

First of all, it is recommended to use high-definition video cameras which support multiple protocols access, network control, H.265/H.264/MJPEG video compression algorithm and dual stream technology. At the same time, due to the complex environment of the project site, the camera should meet the international IP66 standard, and have windproof, rainproof, dustproof and even lightning protection function[2].In addition, in order to meet the needs of night construction on the project site, infrared cameras should be selected.

\subsubsection{Wireless AP}

5.8G wireless AP overcomes the limitation of traditional 2.4G which can only transmit a few hundred meters, and the "'back to back" relay can make multiple devices to build a multi hop link, which overcomes the shortcomings of a lot of equipment that require a non-blocking environment for point-to-point transmission. Therefore, it is more suitable for more complex outdoor environment, the best transmission distance of up to 3 kilometers, the maximum transmission rate of $300 \mathrm{Mbps}$. So it has excellent long-distance transmission performance and can transmit 
multi-channel high-definition video.

\subsubsection{Network Video Recorder}

NVR is an important device that can access network cameras that comply with ONVIF, PSIA, RTSP, ARP protocols and many major vendor protocols; support for IPC plug-and-play; support for preview, storage and playback of HD network video; Management, including IPC parameter configuration, import / export information; support for HDMI output; support for intelligent search, playback and backup; support for important video file locking protection.

\subsection{Transmission method}

\subsubsection{Transmission method of construction site}

For the scale is not large, the construction area is relatively centralized and easy to wiring engineering site, wired network is recommended. For construction site conditions are relatively bad and laying cable network is difficult, 5.8G wireless network is suitable for the transmission of video information.

\subsubsection{Remote network transmission method}

For the remote transmission, priority is given to the use of broadband Internet, because this way is less costly and more stable. But for some remote sites that do not have access to the Internet for the time being, the 3G/4G wireless network can also be used. However, this method is charged according to the flow, so the cost of transmitting video information is higher[3]. In addition, for some construction site with confidentiality requirements, a dedicated optical fiber network can be leased to communicate with carriers for remote transmission.

\section{Function Analysis}

(1) The system can appropriately reduce the number of on-site management personnel, detect violations in time, make sure that the rectification information is communicated and implemented, and improve the efficiency and accuracy of mastering the scene. At the same time through macro-monitoring, construction site layout can also be optimized, and comprehensive deployment the manpower and resources[4].

(2)The system can promote the continuous improvement of the staff in the construction site. The monitoring is equivalent to the electronic eye, and can monitor the construction progress in a whole process and in various directions. For workers, the system can increases its binding force invisible, enhance their sense of responsibility, promote its efforts to learn and master the construction standards and procedures, improve the safety and quality awareness, and ensure the project construction and operation standardization. In addition, the construction process is stored and backup by video. It can check the monitoring information at any time, and also identify the cause of the accident for the first time after the accident occurs, and clarify the accident liability.

(3) The system can monitor the safety production measures on the construction site in real time and effectively monitor the safety elements such as construction machinery, safety net and construction workers' safety protection equipment on the construction operation surface[5]. The system has also realized the digitalization of the work safety protection work, so that the illegal operations and the hidden dangers of security are nowhere to be hidden.

(4) Construction site has many large construction machinery (such as construction elevator, tower crane, etc.). The operation of this kind of machinery has strong technical and professional, without special training, it is difficult for the general people to master. The improper operation of this kind of equipment can easily lead to the occurrence of safety accidents. The system can monitor the operation of the field equipment in real time, and once it finds out the violation, immediately notify the rectification.

(5) Theft of production materials and equipment is also one of the difficulties in site management. 
Some criminals use the construction site management loopholes frequently committing crimes. Not only cause serious losses to the construction enterprises, but also bring security risks to the surrounding residents. Project site equipment remote video monitoring, on-site personnel and equipment import have been fully recorded, through monitoring and video playback will be able to easily find the criminal activities, and the video will be submitted as evidence in judicial departments. This will not only reduce the loss of equipment and materials, but also greatly reduce the crime rate of various crimes, so that the construction unit has a good working environment, improve the quality of construction.

(6)Compared with the long-term use of human resources, the system has high cost performance and can be used repeatedly. The maintenance cost of the system is also small, and it has a solid promotion effect on promoting the construction enterprises to save human resources.

\section{Conclusion}

The application of remote video monitoring system to engineering field control is the promotion of many technologies such as network technology, video surveillance technology in the field of engineering construction. It breaks the limitation of time and region, improves the speed of management process and expedite information exchange, so as to improve the management level and efficiency of the enterprise. It also accelerates the construction of enterprise information, integrates information technology and management processes, and enables information network to become the carrier of information exchange in engineering management, so as to further improve the efficiency of project management.

\section{Acknowledgement}

This paper was funded by the National Natural Science Found of China (No.71171081) and the Beijing Natural Science Foundation (No.9162014)

\section{References}

[1] Pu,xiaoping. Research on safety management and countermeasures of construction enterp- rises [M]. Chang’an University.2012:18.

[2] Hao xiaoyin. Design and application of remote video surveillance system on site Project[J]. construction science and technology, 2016, (12):116-117.

[3] Hong jin. Application of remote video surveillance system in construction projects[J]. Construction Materials \& Decoration , 2016, (31): 157-158.

[4] Han jiajie, Qi ligang, Yang fan, et al. The remote intelligent monitoring system in the application and research of large public construction projects [J].Tianjin construction science and technology, 2017,(2):32-33.

[5] Liu wei.Internet plus monitoring system project of remote management of [A]. Construction technology and Management Symposium [C]. 2016:213-214. 